\title{
Attracting shallow donors: Hydrogen passivation in (Al,Ga,In)-doped $\mathrm{ZnO}$
}

\author{
M. Matsubara, ${ }^{*}$ M. N. Amini, ${ }^{*}$ R. Saniz, D. Lamoen, and B. Partoens \\ CMT \& EMAT, Departement Fysica, Universiteit Antwerpen, Groenenborgerlaan 171, B-2020 Antwerpen, Belgium
}

(Received 24 November 2011; published 22 October 2012)

\begin{abstract}
The hydrogen interstitial and the substitutional $\mathrm{Al}_{\mathrm{Zn}}, \mathrm{Ga}_{\mathrm{Zn}}$, and $\mathrm{In}_{\mathrm{Zn}}$ are all shallow donors in $\mathrm{ZnO}$ and lead to $n$-type conductivity. Although shallow donors are expected to repel each other, we show by first-principles calculations that in $\mathrm{ZnO}$ these shallow donor impurities attract and form a complex, leading to a donor level deep in the band gap. This puts a limit on the $n$-type conductivity of (Al,Ga,In)-doped $\mathrm{ZnO}$ in the presence of hydrogen.
\end{abstract}

DOI: 10.1103/PhysRevB.86.165207

PACS number(s): 71.55.Gs, 61.72.-y, 71.15.Mb, 71.20.Mq

\section{INTRODUCTION}

The incorporation of hydrogen in a material can strongly affect its electronic properties. Furthermore, its behavior depends on the host into which it is introduced. Mostly, interstitial hydrogen is amphoteric, i.e., it can act either as a donor $\left(\mathrm{H}_{i}^{+}\right)$or an acceptor $\left(\mathrm{H}_{i}^{-}\right){ }^{1}$ Which of these two prevails depends on the Fermi level: it acts as a donor in $p$-type materials and as an acceptor in $n$-type materials. Consequently, it counteracts the conductivity caused by extrinsic dopants. It is important to realize that this passivation of extrinsic dopants by hydrogen is not caused by the formation of a neutral complex. The formation of such a neutral complex may just be the consequence after compensation, i.e., after the trapping of a free hole or electron by hydrogen. Afterwards, the positively charged $\mathrm{H}$ and negatively charged acceptor, or negatively charged $\mathrm{H}$ and positively charged donor, attract and may form a neutral complex.

In $\mathrm{ZnO}$, however, the behavior of hydrogen is not amphoteric. ${ }^{1}$ Nominally undoped $\mathrm{ZnO}$ shows $n$-type conductivity and it was first predicted by first-principles calculations based on density functional theory, ${ }^{2}$ and later experimentally verified, ${ }^{3}$ that interstitial hydrogen acts as a shallow donor in $\mathrm{ZnO}$ and can cause this $n$-type behavior.

$\mathrm{ZnO}$ is a wide-band-gap $\left(\sim 3.4 \mathrm{eV}\right.$ at room temperature $\left.{ }^{4}\right)$ semiconductor with many possibilities for technological applications as a transparent conductor in solar cells, ${ }^{5}$ flat panel displays, ${ }^{6}$ etc. It is well known that the electronic conductivity of $\mathrm{ZnO}$ can be dramatically improved by doping it with group III elements $\mathrm{Al}, \mathrm{Ga}$, or $\mathrm{In}^{7}$ This makes $\mathrm{ZnO}$ an alternative for indium tin oxide, which shows highest conductivity among transparent conducting oxides but is less abundant and therefore more expensive. It is then also important to know what limits the conductivity of $\mathrm{ZnO}$ doped by $\mathrm{Al}, \mathrm{Ga}$, or In. Properties such as formation energy, energy band structure and equilibrium geometry of $\mathrm{Al}, \mathrm{Ga}$, and In doped $\mathrm{ZnO}$ were studied systematically and it was shown that all three dopants are indeed shallow donors and that they possess the intrinsic qualities to be good $n$-type transparent conducting oxides. ${ }^{8}$

As the concentration becomes high, the interaction between defects becomes unavoidable. Because it is very difficult to remove hydrogen from the crystal growth environment, it is then also natural to ask what the influence is of interstitial hydrogen on $\mathrm{Al}, \mathrm{Ga}$, or In doped $\mathrm{ZnO}$. Recently a number of experiments were reported where both $\mathrm{H}$ and group III elements are codoped into $\mathrm{ZnO}$. It was shown that the conductivity of $\mathrm{ZnO}$ codoped with $\mathrm{H}$ and group III elements is better than that of $\mathrm{ZnO}$ doped with group III elements up to certain amount of $\mathrm{H}$ inclusion. ${ }^{9}$

Apart from single hydrogen dopants, the influence of hydrogen on doped $\mathrm{ZnO}$ structures has been studied before by first-principles calculations. It was found that hydrogen passivates nitrogen dopants, ${ }^{10}$ silver dopants, ${ }^{11}$ and $\mathrm{Zn}$ vacancies ${ }^{12}$ in $\mathrm{ZnO}$, and forms complexes. This is not very surprising, as in all these cases, hydrogen, which is a (positive) shallow donor, is attracted to a (negative) acceptor impurity. This is not unique to hydrogen. Also donor-like $\mathrm{As}_{\mathrm{Zn}}$ and acceptor-like $\mathrm{V}_{\mathrm{Zn}}$ attract each other and form a complex in As doped $\mathrm{ZnO} .{ }^{13}$ Recently, also the attractive interaction between two donor impurities was predicted by first-principles studies in $\mathrm{ZnO}$, namely the deep donor $\mathrm{V}_{\mathrm{O}}$ (the oxygen vacancy) and the shallow donor $\mathrm{Zn}_{i}{ }^{14}$ (the $\mathrm{Zn}$ interstitial). In this work, we consider the two shallow donors interstitial hydrogen $\mathrm{H}_{i}$ and substitutional $X_{\mathrm{Zn}}$ with $X=\mathrm{Al}$, Ga, or In.

\section{METHODS}

We performed density functional theory calculations as implemented in the Vienna ab initio simulation package (VASP) code. ${ }^{15,16}$ In order to examine interactions between defects, a large supercell consisting of 192 atoms was considered. ${ }^{17}$ A single $\mathrm{Al}, \mathrm{Ga}$, or In atom was chosen to substitute a $\mathrm{Zn}$ atom, while for the $\mathrm{H}$ atom an extensive number of positions around the group III element were considered. For all these configurations the formation energies for different charge states were calculated using the method of Ref. 18, which combines local-density approximation (LDA) and LDA $+U^{19}$ calculations with an extrapolation scheme to overcome the band-gap problem. This method was recently confirmed to give reliable results for the transition energies between different charge states of the impurity, both for $\mathrm{ZnO}$ with interstitial $\mathrm{H}^{20}$ as for $\mathrm{ZnO}$ doped with a group III element, ${ }^{8}$ by comparing the results with hybrid functional calculations.

\section{RESULTS AND DISCUSSION}

As we will compare the formation energies of the defect complexes with the corresponding isolated defects, we first considered the individual interstitial hydrogen $\mathrm{H}_{i}$ and the substitutional $X_{\mathrm{Zn}}$ (with $X=\mathrm{Al}$, Ga, or In) defects. As expected, the +1 charge state of the substitutional $X_{\mathrm{Zn}}$ defect is the ground state for all values of the Fermi energy in 


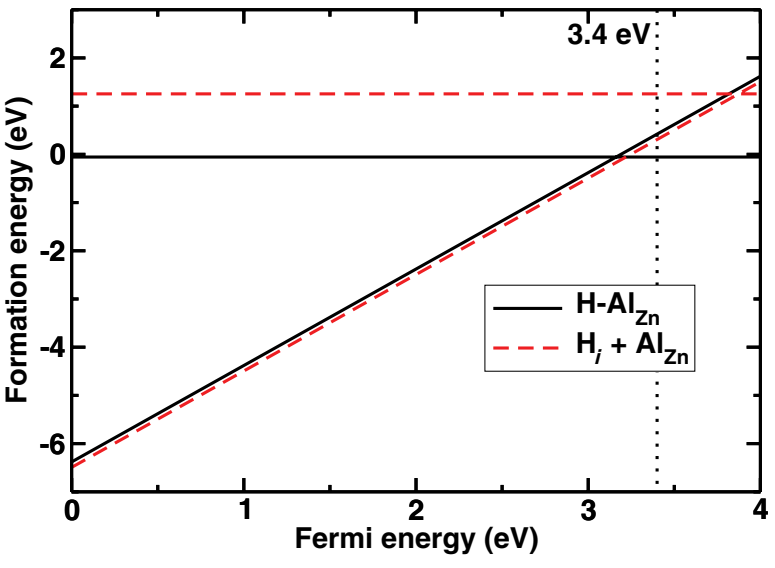

FIG. 1. (Color online) Formation energy as function of Fermi energy for the $\mathrm{H}-\mathrm{Al}_{\mathrm{Zn}}$ complex (full black curve) and for two noninteracting interstitial hydrogen $\mathrm{H}_{i}$ and substitutional $\mathrm{Al}_{\mathrm{Zn}}$ impurities (dashed red curve), in the oxygen-poor limit.

the band gap. The results are presented in Ref. 8. For the interstitial hydrogen $\mathrm{H}_{i}$ defect, different positions are possible. Our calculations confirm that the bond center position (labeled $\mathrm{BC}_{\|}$, according to Ref. 2), with the $\mathrm{H}$ atom positioned between a $\mathrm{Zn}$ and an $\mathrm{O}$ atom is the preferential interstitial position. Furthermore, our formation energies are in agreement with the results presented in Ref. 21.

Let us now consider the defect complexes. As for the isolated interstitial hydrogen $\mathrm{H}_{i}$ defect, we considered many different positions, and we find that, for all charge states, the lowest formation energy is obtained when the $\mathrm{H}$ atom is located close to the octahedral interstitial position next to an $\mathrm{Al}, \mathrm{Ga}$, or In atom (in the notation defined in Ref. 2, this position can be called the antibonding $\mathrm{AB}_{\mathrm{Al}, \perp}$ configuration). Note that this position differs from the isolated $\mathrm{H}_{i}$ defect. We can now compare the formation energies for two noninteracting impurities, obtained by adding the formation energy of a single interstitial $\mathrm{H}$ atom $\left(\mathrm{H}_{i}\right)$ at the $\mathrm{BC}_{\|}$position and a single $\mathrm{Al}$, $\mathrm{Ga}$, or In dopant in $\mathrm{ZnO}\left(X_{\mathrm{Zn}}\right)$, with the formation energy of the defect complexes, with the $\mathrm{H}$ atom at the $\mathrm{AB}_{\mathrm{Al}, \perp}$ position. The results are shown in Figs. 1, 2 and 3 for the codoping cases $\mathrm{H}-\mathrm{Al}, \mathrm{H}-\mathrm{Ga}$, and $\mathrm{H}-\mathrm{In}$, respectively. The shown curves are for the oxygen-poor limit. For the oxygen-rich limit, all curves

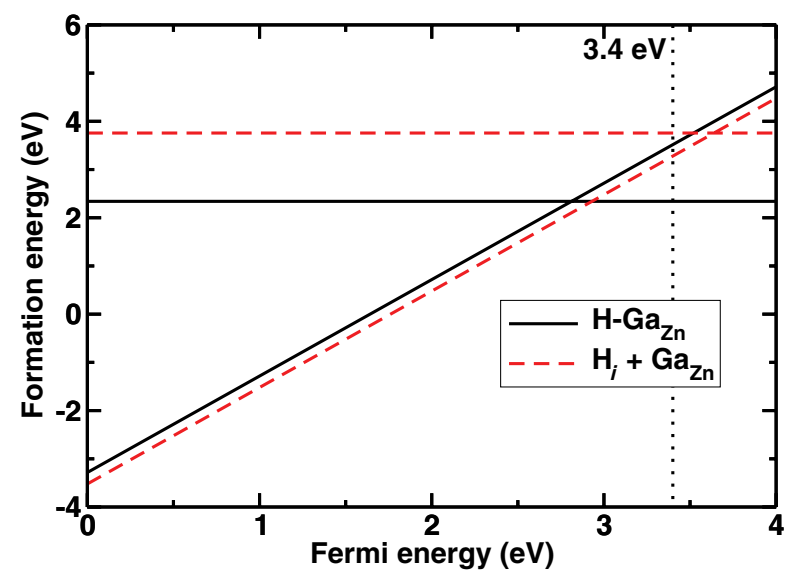

FIG. 2. (Color online) As in Fig. 1, but for Ga-H codoped ZnO.

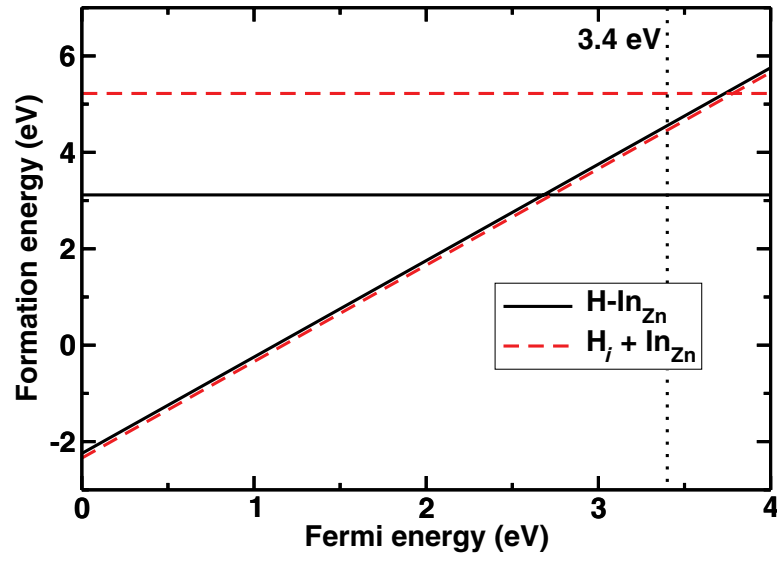

FIG. 3. (Color online) As in Fig. 1, but for In-H codoped $\mathrm{ZnO}$.

shift down by $3.43 \mathrm{eV}$. This choice has no influence on our conclusions, as they are only based on the crossing points between the formation energy curves for different charge states. As both $\mathrm{H}$ and (Al, Ga, or In) can give away a single electron, the +2 charge state is the ground state for small values of the Fermi energy. (The +1 case is not shown, as it never becomes the ground state.) It is clear that the case of two noninteracting, thus infinitely separated, impurities has the lowest formation energy. In this +2 charge state, both shallow donors repel each other. More importantly, however, for Fermi-energy values close to the band gap of $3.4 \mathrm{eV}$, the neutral $\mathrm{H}-(\mathrm{Al}, \mathrm{Ga}, \mathrm{In})$ complex has the lowest formation energy. Furthermore, the crossing between the noninteracting charge case +2 and the neutral complex occurs in the band gap, i.e., a deep level is formed. This crossing occurs at 0.18, 0.47, and $0.67 \mathrm{eV}$ below the bottom of the conduction band for $\mathrm{Al}-\mathrm{H}, \mathrm{Ga}-\mathrm{H}$, and $\mathrm{In}-\mathrm{H}$, respectively. This is a consequence of the large decrease in formation energy of the neutral complex in comparison to the formation energy of two noninteracting neutral impurities: $1.31 \mathrm{eV}$ for $\mathrm{Al}-\mathrm{H}, 1.42 \mathrm{eV}$ for $\mathrm{Ga}-\mathrm{H}$, and $2.11 \mathrm{eV}$ for $\mathrm{In}-\mathrm{H}$. As a consequence, the shallow donor $\mathrm{H}$ binds to and passivates the shallow donor (Al, Ga or In).

The formation of a deep level is also clear from the density of states (DOS). As an example, the DOS for the Al-H codoped case obtained within the LDA approximation is shown in Fig. 4. The neutral complex is considered in its ground-state configuration. Comparing Figs. 4(a) and 4(b) for the pure $\mathrm{ZnO}$ crystal and the codoped case, a sharp peak appears in the $\mathrm{ZnO}$ band gap. From the projected (local) DOS plots on H [Fig. 4(c)] and $\mathrm{Al}$ [Fig. 4(d)] we observe that this deep donor level has $\mathrm{H} s$ and $\mathrm{Al} p$ character. While an individual interstitial $\mathrm{H}_{\mathrm{i}}$ and a substitutional $\mathrm{Al}_{\mathrm{Zn}}$ atom in $\mathrm{ZnO}$ have both a defect level in the conduction band with these characters, a bonding level is formed in the case where they form a complex, which lowers the energy to a value in the band gap.

To gain a better understanding of the physics behind this finding, we considered the bond length in the neutral complex. The bond between both shallow donors is clearly reflected in the interatomic distances. In the neutral case, the equilibrium distance between the interstitial $\mathrm{H}$ atom and the substitutional group III atom is $1.86 \AA$ for the $\mathrm{Al}$ case, $1.77 \AA$ for the Ga case, and $1.87 \AA$ for the In case. However, in the +2 case, these distances increase till close to $3.15 \AA$ for all three 


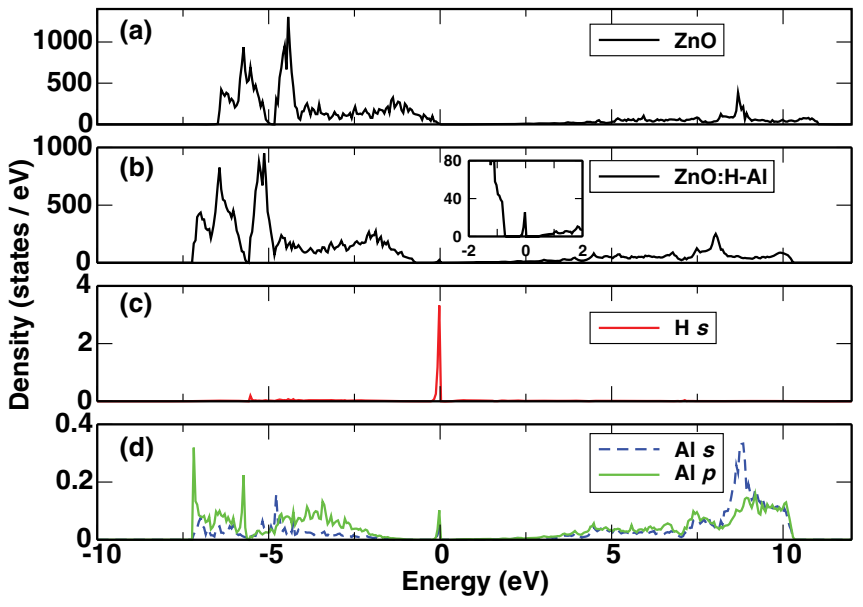

FIG. 4. (Color online) Calculated DOS, with zero energy taken at the Fermi level: (a) total DOS for the pure $\mathrm{ZnO}$ crystal; (b) total DOS for the Al-H codoped $\mathrm{ZnO}$, where the inset is a zoom around the Fermi level; (c) projected (local) DOS on the H site; (d) projected (local) DOS on the Al site.

cases, showing a clear repulsion. As an example, for the $\mathrm{Al}-\mathrm{H}$ codoped $\mathrm{ZnO}$, the structure of the neutral $\mathrm{Al}-\mathrm{H}$ complex is shown in Figs. 5(a) and 5(b), and for the repelling +2 case in Figs. 5(c) and 5(d). It is also interesting to compare these bond lengths in the case of the neutral complex with those of the corresponding molecular species $\mathrm{AlH}, \mathrm{GaH}$, and $\mathrm{InH}$, which are $1.65,1.66$, and $1.84 \AA .{ }^{22}$ The $\mathrm{Al}-\mathrm{H}$ and $\mathrm{Ga}-\mathrm{H}$ bond lengths are thus longer in comparison to the length in the molecular species, especially in the Al-H case, while the In-H bond length is much closer to the molecular structure $\mathrm{InH}$. This shows that there is indeed a bond in the neutral H-X complex, although weaker than in the molecular case.

To support this view and to propose an experimental way to detect these complexes, we also studied a vibrational property. The microscopic geometry for hydrogen in semiconductors is usually determined through observation of the infrared spectrum of the related local vibrational mode. In order to facilitate the experimental observation of the $\mathrm{H}-(\mathrm{Al}, \mathrm{Ga}, \mathrm{In})$ complex, we calculate the $\mathrm{H}-X$ stretching mode. As the $\mathrm{H}$ atom is so light we also take into account the anharmonic effect. Therefore the potential curve is fitted by the formula ${ }^{23}$

$$
V(x)=\frac{k}{2} x^{2}+\alpha x^{3}+\beta x^{4} .
$$

We have taken displacements up to $\pm 30 \%$ of the $X$-H bond length along the bond direction. By applying perturbation theory to the one-dimensional Schrödinger equation, an approximated analytical solution in the case of the anharmonic potential [Eq. (1)] is given by ${ }^{24}$

$$
\omega=\omega_{\mathrm{H}}+\Delta \omega_{\mathrm{A}}=\sqrt{\frac{k}{\mu}}-3 \frac{\hbar}{\mu}\left[\frac{5}{2}\left(\frac{\alpha}{k}\right)^{2}-\frac{\beta}{k}\right],
$$

where $\omega$ is the frequency of the mode, $\omega_{H}$ and $\Delta \omega_{A}$ are its harmonic and anharmonic contribution, and $\mu$ is the reduced mass. The obtained results for the frequency of the local
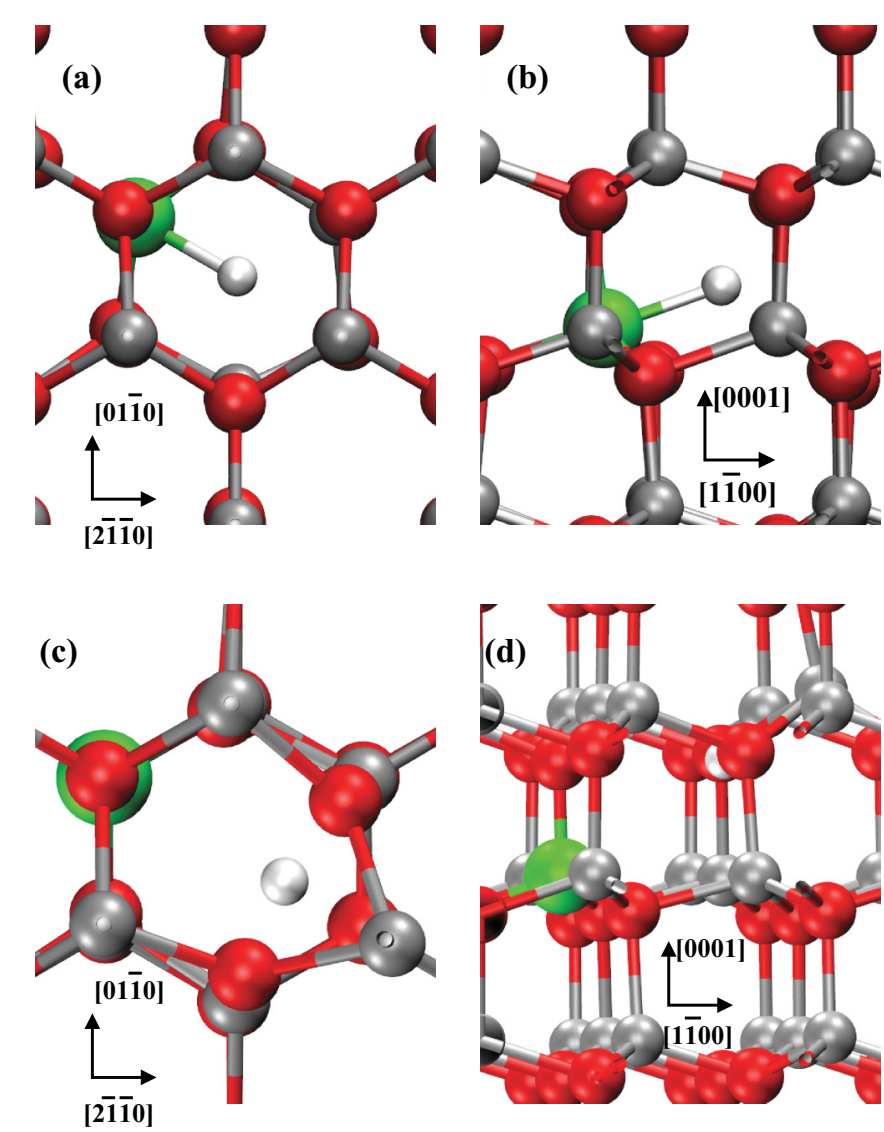

FIG. 5. (Color online) (a) and (b) The Al-H neutral complex. (c), (d) The +2 charge case of the Al-H complex. The red atom is $\mathrm{Zn}$, the grey atom is $\mathrm{O}$, the green atom is $\mathrm{Al}$, and the white atom is $\mathrm{H}$. These figures were generated by VMD version $1.8 .7 .^{25}$

vibrational modes are shown in Table I. Again we can compare with the corresponding frequencies of the molecular species. These results are also included in Table I under $\omega_{\text {mol }}$ (taken from Ref. 22). Note that the obtained frequencies for the complex are smaller than in their molecular equivalents. The difference is most pronounced for $\mathrm{Al}-\mathrm{H}$, and smallest for In-H. The larger the difference, the weaker the bond is in the complex in comparison with the molecular equivalent. This trend is consistent with the trend that was observed for the bond lengths. This then also explains why the defect level of the complex is deepest in the In-H case.

TABLE I. Calculated harmonic frequencies $\left(\omega_{H}\right)$, anharmonic contributions $\left(\omega_{A}\right)$, and total frequencies $(\omega)$ of the local vibrational mode of the $X-\mathrm{H}$ complex, and the corresponding frequency $\left(\omega_{\operatorname{mol}}\right)$ in the corresponding molecular structure, taken from Ref. 22. All frequencies are expressed in $\mathrm{cm}^{-1}$.

\begin{tabular}{lcccc}
\hline \hline Complex & $\omega_{H}$ & $\Delta \omega_{A}$ & $\omega$ & $\omega_{\text {mol }}$ (Ref. 22) \\
\hline H-Al & 1266 & -54 & 1212 & 1682 \\
H-Ga & 1265 & -64 & 1201 & 1604 \\
H-In & 1350 & -71 & 1280 & 1475 \\
\hline \hline
\end{tabular}




\section{CONCLUSION}

To summarize, we have studied by first-principles calculations the codoping of $\mathrm{ZnO}$ with hydrogen and a group III element (Al, Ga, or In). While these impurities are known to be shallow donors in $\mathrm{ZnO}$, and are therefore expected to repel each other, we show on the contrary that they attract each other, resulting in a neutral complex which forms a deep level. Hydrogen thus passivates the group III impurity in $\mathrm{ZnO}$, which puts a limit on its $n$-type conductivity. In semiconductors in which hydrogen shows amphoteric behavior, passivation of a shallow impurity does not mean that a complex is formed; the formation of such a complex may just be a consequence of the passivation. In $\mathrm{ZnO}$ however, in which hydrogen acts as a shallow donor, it is the pairing between hydrogen and the substitutional shallow donor into a neutral complex that realizes the passivation.

\section{ACKNOWLEDGMENTS}

We gratefully acknowledge financial support from the IWT-Vlaanderen through the ISIMADE project, the FWOVlaanderen through project G.0191.08, and the BOF-NOI of the University of Antwerp. This work was carried out using the HPC infrastructure of the University of Antwerp (CalcUA) a division of the Flemish Supercomputer Center VSC, which is funded by the Hercules foundation. M.M. acknowledges financial support from the GOA project "XANES meets ELNES" of the University of Antwerp.
*M. Matsubara and M. N. Amini contributed equally to this work.

${ }^{1}$ C. G. Van de Walle and J. Neugebauer, Nature (London) 423, 626 (2003).

${ }^{2}$ C. G. Van de Walle, Phys. Rev. Lett. 85, 1012 (2000).

${ }^{3}$ S. F. J. Cox, E. A. Davis, S. P. Cottrell, P. J. C. King, J. S. Lord, J. M. Gil, H. V. Alberto, R. C. Vilão, J. Piroto Duarte, N. Ayres de Campos, A. Weidinger, R. L. Lichti, and S. J. C. Irvine, Phys. Rev. Lett. 86, 2601 (2001); D. M. Hofmann, A. Hofstaetter, F. Leiter, H. Zhou, F. Henecker, B. K. Meyer, S. B. Orlinskii, J. Schmidt, and P. G. Baranov, ibid. 88, 045504 (2002).

${ }^{4}$ Y. Chen, D. M. Bagnall, H.-J. Koh, K.-T. Park, K. Hiraga, Z. Zhu, and T. Yao, J. Appl. Phys. 84, 3912 (1998).

${ }^{5}$ A. Nuruddin and J. R. Abelson, Thin Solid Films 394, 48 (2001).

${ }^{6}$ R. G. Gordon, MRS Bull. 25, 52 (2000).

${ }^{7}$ T. Minami, MRS Bull. 25, 38 (2000).

${ }^{8}$ R. Saniz, Y. Xu, M. Matsubara, M. N. Amini, H. Dixit, D. Lamoen, and B. Partoens, J. Phys. Chem. Solids 74, 45 (2013).

${ }^{9}$ W. Liu, G. Du, Y. Sun, Y. Xu, T. Yang, X. Wang, Y. Chang, and F. Qiu, Thin Solid Films 515, 3057 (2007); S. H. Lee, T. S. Lee, K. S. Lee, B. Cheong, Y. D. Kim, and W. M. Kim, J. Phys. D: Appl. Phys. 41, 095303 (2008); J. Electroceram. 23, 468 (2009); S. J. Tark, Y.-W. Ok, M. G. Kang, H. J. Lim, W. M. Kim, and D. Kim, ibid. 23, 548 (2009); D.-H. Kim, S.-H. Lee, G.-H. Lee, H.-B. Kim, K. H. Kim, Y.-G. Lee, and T.-H. Yu, J. Appl. Phys. 108, 023520 (2010).

${ }^{10}$ X. N. Li, B. Keyes, S. Asher, S. B. Zhang, S.-H. Wei, T. J. Coutts, S. Limpijumnong, and C. G. Van de Walle, Appl. Phys. Lett. 86, 122107 (2005).
${ }^{11}$ H. Y. He, J. Hu, and B. C. Pan, J. Chem. Phys. 130, 204516 (2009).

${ }^{12}$ S. Z. Karazhanov, E. S. Marstein, and A. Holt, J. Appl. Phys. 105, 033712 (2009).

${ }^{13}$ S. Limpijumnong, S. B. Zhang, S.-H. Wei, and C. H. Park, Phys. Rev. Lett. 92, 155504 (2004).

${ }^{14}$ Y.-S. Kim and C. H. Park, Phys. Rev. Lett. 102, 086403 (2009).

${ }^{15}$ G. Kresse and J. Furthmüller, Phys. Rev. B 54, 11169 (1996).

${ }^{16}$ G. Kresse and D. Joubert, Phys. Rev. B 59, 1758 (1999).

${ }^{17}$ Our supercell consists of 192 atoms $(4 \times 4 \times 3$ unit cells). Wave functions are expanded with plane-wave basis sets up to a cutoff energy of $400 \mathrm{eV}$ and the Brillouin zone is sampled with the $\Gamma$ centered $k$-point grids of $2 \times 2 \times 2$ containing six special $k$ points. Optimization of ionic positions is allowed to proceed until the largest force component is less than $0.01 \mathrm{eV} / \AA$.

${ }^{18}$ A. Janotti and C. G. Van de Walle, Phys. Rev. B 76, 165202 (2007).

${ }^{19} \mathrm{We}$ have chosen $U=4.7 \mathrm{eV}$, as obtained in A. Janotti, D. Segev and C. G. Van de Walle, Phys. Rev. B 74, 045202 (2006).

${ }^{20}$ A. Janotti and C. G. Van de Walle, Phys. Status Solidi B 248, 799 (2011).

${ }^{21}$ A. Janotti and C. G. Van de Walle, Nat. Mater. 6, 44 (2007).

${ }^{22}$ S. Aldridge and A. J. Downs, Chemical Reviews 101, 3305 (2001).

${ }^{23}$ S. Limpijumnong, J. E. Northrup, and C. G. Van de Walle, Phys. Rev. B 68, 075206 (2003).

${ }^{24}$ L. D. Landau and E. M. Lifshitz, in Quantum Mechanics NonRelativistic Theory, 3rd. ed., Chap. 6 (Butterworth-Heinemann, Boston, 1981), p. 136.

${ }^{25}$ W. Humphrey, A. Dalke, and K. Schulten, J. Mol. Graphics 14, 33 (1996). 